\title{
Detection of Pneumocystis carinii DNA by PCR amplification in various rat organs in experimental pneumocystosis
}

\author{
M. RABODONIRINA, R. WILMOTTE**, E. DANNAOUI, F. PERSAT, G. BAYLE and M. MOJON†
}

Département de Parasitologie et de Mycologie, Université Claude-Bernard, 8 avenue Rockefeller, 69373 Lyon Cedex 08 and *Génétique Moléculaire Humaine, CNRS URA 1171, Institut Pasteur de Lyon, Avenue Tony Garnier, 69365 Lyon Cedex 07, France

\begin{abstract}
Pneumocystosis is usually a disease of the lungs, but the number of cases of extrapulmonary pneumocystosis has greatly increased during the AIDS epidemic. Much remains unknown about the frequency and mechanisms of dissemination. In the present study, a systematic search for Pneumocystis carinii by PCR with primers specific for mitochondrial rRNA was performed in the lung, liver, spleen and kidney of 12 immunosuppressed rats and two immunocompetent rats. The amplified products were analysed by Southern hybridisation with a digoxigenin-11-dUTP labeled probe. $P$. carinii DNA was found in lungs in all 14 rats and in at least one organ other than lung in 11 immunosuppressed rats and the two control rats. We suggest that extrapulmonary dissemination may not be an exceptional phenomenon in the course of pneumocystosis, but rather part of the natural evolution of the disease.
\end{abstract}

\section{Introduction}

Pneumocystis carinii pneumonia is a major cause of morbidity and mortality in HIV-infected patients. Recently, an increasing number of cases of extrapulmonary and disseminated pneumocystosis, or both, has been reported in AIDS patients [1]. This raises issues about the natural history of the disease; what is the frequency of the dissemination; is this dissemination always associated with a pulmonary infection; and does the proliferation occur only in the immunosuppressed host or does $P$. carinii proliferate in extrapulmonary sites in the normal host? A few experimental studies on extra-pulmonary dissemination of $P$. carinii have been done $[2,3]$. Involvement of various organs has been observed in some cases, but the number of $P$. carinii organisms seems to be very low in sites other than lung. Therefore, a very sensitive technique is required to detect $P$. carinii in these sites. Recently, the polymerase chain reaction (PCR) has been reported as a specific diagnostic method for $P$. carinii pneumonia [4-9]. The results of various studies indicate that PCR is more sensitive than conventional methods of diagnosis (staining methods or immuno-

Received 13 Sept. 1996; revised version accepted 29 Dec. 1996.

Corresponding author: Dr M. Rabodonirina.

†Deceased: this paper is dedicated to her memory. fluorescence assays) for the detection of $P$. carinii in human samples. Thus, the present study compared PCR to Giemsa and methenamine silver stains in a systematic search for $P$. carinii in the lung and in various organs of untreated control rats and immunosuppressed rats.

\section{Materials and methods \\ Experimental animals}

Fourteen female Sprague-Dawley rats (Iffa Credo, Lyon, France), all weighing c. $200 \mathrm{~g}$ were used. Twelve rats, housed in isolation cages, were provided with drinking water containing dexamethasone $(2 \mathrm{mg} / \mathrm{L})$ [10], to induce immunosuppression, and tetracycline $(500 \mathrm{mg} / \mathrm{L})$ to prevent bacterial infections. The remaining two rats, caged in the same room as the immunosuppressed rats, did not receive dexamethasone and served as sentinel controls. All the rats were fed a normal protein diet. Twelve weeks after the beginning of immunosuppression the mean weight of the control rats was $310 \mathrm{~g}$ (range $300-320 \mathrm{~g}$ ). The mean weight of the immunosuppressed rats was $163 \mathrm{~g}$ (range 150$180 \mathrm{~g}$ ) and they showed clinical signs of severe infection (hair loss, shivering, dyspnoea). At this time the rats were anaesthetised with ether and exsanguinated by intracardiac puncture. Lungs, liver, spleen and kidney were collected with extreme care to avoid cross- 
contamination between tissues by using separate surgical materials. Organs were stored at $-80^{\circ} \mathrm{C}$. Smears prepared from each organ were stained with Giemsa and methenamine silver methods.

\section{$D N A$ preparation}

To avoid cross-contamination of the extra-pulmonary tissues with infected lung tissues, the other tissues were processed several weeks after lung tissues and new materials and solutions were used. Tissue samples of each organ $(0.25 \mathrm{~g})$ were homogenised in separate Waring blenders and washed three times in phosphatebuffered saline (PBS) ( $\mathrm{pH}$ 7.2) to remove as much blood as possible. The blenders were cleaned and autoclaved each time before use. The homogenates were incubated at $55^{\circ} \mathrm{C}$ overnight in a solution containing $50 \mathrm{mM} \mathrm{KCl}, 10 \mathrm{mM}$ Tris- $\mathrm{HCl}$ (pH 8.3), $2.5 \mathrm{mM} \mathrm{MgCl}_{2}$, gelatin $0.1 \mathrm{mg} / \mathrm{ml}$, Nonidet $\mathrm{P}-40$ $0.45 \%$, Tween-20 $0.45 \%$ and proteinase $\mathrm{K}$ at a final concentration of $60 \mu \mathrm{g} / \mathrm{ml}$. DNA was extracted with phenol-chloroform, precipitated with ethanol, and dissolved in water.

\section{DNA amplification and hybridisation}

The primers described by Wakefield et al. [10] were used for amplification of part of the mitochondrial gene encoding the large subunit of rRNA: pAZ102-E (5'GATGGCTGTTTCCAAGCCCA-3') and pAZ102-H (5'-GTGTACGTTGCAAAGTACTC-3'). One $\mu \mathrm{g}$ of DNA extracted from each organ was amplified in a reaction mixture $(100 \mu \mathrm{l})$ containing $50 \mathrm{mM} \mathrm{KCl}$, $10 \mathrm{mM}$ Tris- $\mathrm{HCl}$ ( $\mathrm{pH} 8.3$ ), $1.5 \mathrm{mM} \mathrm{MgCl}_{2}$, gelatin $0.01 \%, 0.25 \mathrm{mM}$ each of dATP, dTTP, dCTP and dGTP, 100 pmol each of the oligonucleotide primers and 2.5 units of Taq-polymerase (Perkin Elmer Cetus, Norwalk, CT, USA). After an initial 5-min denaturation step, DNA was amplified for 40 cycles in a Trio-Thermoblock thermocycler (Biometra, Göttingen, Germany). Each cycle consisted of $1.5 \mathrm{~min}$ denaturation at $94^{\circ} \mathrm{C}$, $1 \mathrm{~min}$ annealing at $56^{\circ} \mathrm{C}$ and $1 \mathrm{~min}$ extension at $72^{\circ} \mathrm{C}$. The amplification of lung tissues took place on different days from those on which the other tissues were amplified. Standard protocols to avoid contamination were followed [11], i.e., DNA extraction and amplification procedures were performed in separate rooms within the laboratory and aerosol-barrier pipette tips were used for all reagent transfers and negative controls (water) were run in each experiment. The amplified products (355 bp) were electrophoresed in agarose $1.5 \%$ gels and visualised after ethidium bromide staining. They were then Southern-blotted on to a Hybond $\mathrm{N}^{+}$nylon membrane (Amersham), and hybridised with an internal oligonucleotide probe, pAZ102-L1 (5'-ATAAGGTGAGGAGTCGAGAG-3') [10]. The probe was labelled with digoxigenin-11dUTP by a non-radioactive DNA labelling kit (Boehringer Mannheim, Mannheim, Germany). The hybrids were detected by enzyme-linked immunoassay.

\section{Sensitivity of the $P C R$}

To determine the level of detection of the PCR, a 20- $\mu 1$ sample of a human bronchoalveolar lavage (BAL) specimen known to contain $P$. carinii was placed on a microscope slide. The smears were stained with Giemsa and methenamine silver. PCR was performed as described above on another $10-\mu 1$ sample of the same BAL specimen and on 10-fold serial dilutions (1 in $10^{1}-1$ in $10^{10}$ ) of this sample. The amplification products were visualised on an agarose $1.5 \%$ gel containing ethidium bromide.

\section{Results and Discussion}

P. carinii DNA was detected by PCR in the lungs of all 14 rats and in at least one other organ in 13 of them (Table 1). The results for the kidney are particularly interesting as $P$. carinii DNA was found in this tissue in the two control rats (Fig. 1). On the contrary, stains failed to show the presence of $P$. carinii organisms in any specimens from the two immunocompetent rats. In the 12 immunosuppressed rats, $P$. carinii trophozoites were observed on the Giemsa-stained smears prepared from nine lungs and one liver. Cysts were detected by methenamine silver stain in the smears prepared from 10 lungs, two spleens, one liver and one kidney (Table 1). Thus we have confirmed by PCR the healthy carrier state of the sentinel control rats exposed to $P$. carinii infected-rats, as previously described by Sepkowitz et al. [12]. These results suggest almost systematic extra-pulmonary dissemination of $P$. carinii to several organs in both the immunosuppressed and

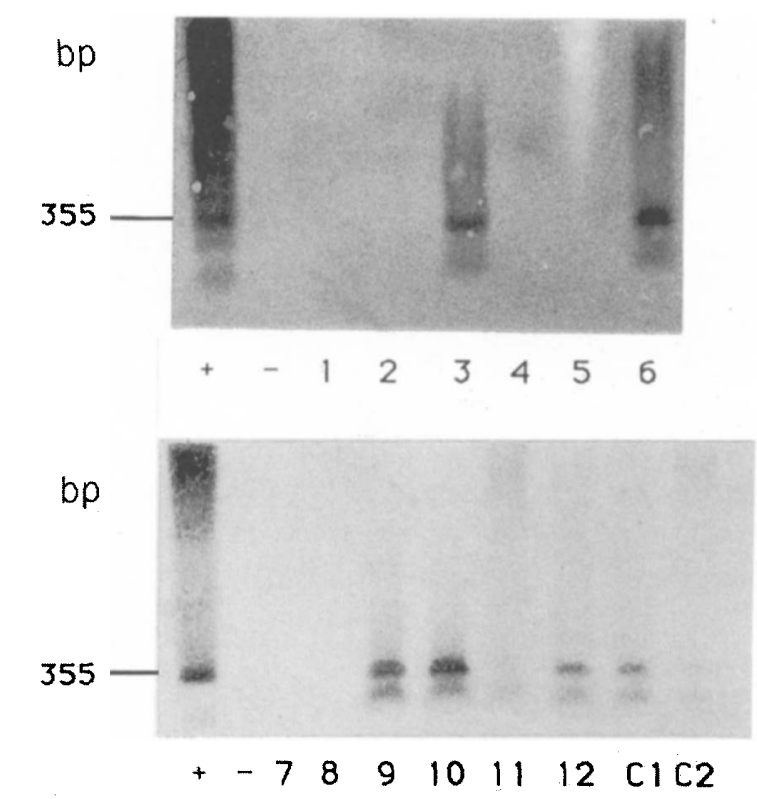

Fig. 1. PCR detection of $P$. carinii DNA in the kidney of 12 immunosuppressed rats and two control rats. Southern hybridisation of electrophoresis gel with digoxigenin-11dUTP-labelled oligonucleotide probe; + , positive control ( $P$. carinii DNA from rat lung); -, negative control (water). The immunosuppressed rats are numbered 1-12 and the two control rats are numbered $\mathrm{C} 1$ and $\mathrm{C} 2$. 
Table 1. Detection of $P$. carinii by Giemsa stain (GS), methenamine silver stain (MSS) and PCR in the 12 immunosuppressed rats and the two sentinel control rats

\begin{tabular}{|c|c|c|c|c|c|c|c|c|c|c|c|c|}
\hline \multirow{2}{*}{ Rat no. } & \multicolumn{3}{|c|}{ Lung } & \multicolumn{3}{|c|}{ Spleen } & \multicolumn{3}{|c|}{ Liver } & \multicolumn{3}{|c|}{ Kidney } \\
\hline & GS & MSS & PCR & GS & MSS & PCR & GS & MSS & PCR & GS & MSS & PCR \\
\hline 1 & + & + & + & - & - & + & - & - & + & - & - & - \\
\hline 2 & - & - & + & - & - & + & - & - & - & - & - & - \\
\hline 3 & + & + & + & - & + & + & + & + & + & - & + & + \\
\hline 4 & - & + & + & - & - & + & - & - & + & - & - & - \\
\hline 5 & + & + & + & - & - & - & - & - & + & - & - & - \\
\hline 6 & + & + & + & - & - & + & - & - & + & - & - & + \\
\hline 7 & - & - & + & - & - & - & - & - & - & - & - & - \\
\hline 8 & + & + & + & - & + & + & - & - & - & - & - & - \\
\hline 9 & + & + & + & - & - & + & - & - & t & - & - & + \\
\hline 10 & + & + & + & - & - & - & - & - & - & - & - & + \\
\hline 11 & + & + & + & - & - & - & - & - & - & - & - & + \\
\hline 12 & + & + & + & - & - & - & - & - & - & - & - & + \\
\hline Control 1 & - & - & + & - & - & + & - & - & - & - & - & + \\
\hline Control 2 & - & - & + & - & - & - & - & - & - & - & - & + \\
\hline Total & 9 & 10 & 14 & 0 & 2 & 8 & 1 & 1 & 6 & 0 & 1 & 8 \\
\hline
\end{tabular}

immunocompetent rats. The present study also confirmed that PCR, in contrast to conventional staining techniques, is able to detect a very low level of infection in various organs. Amplification products were observed after PCR of an undiluted BAL specimen and in specimens diluted 1 in $10^{1}, 1$ in $10^{2}, 1$ in $10^{3}$ and 1 in $10^{4}$. This indicates a limit of detection of 10 organisms (data not shown).

The number of human cases of extra-pulmonary dissemination of $P$. carinii has increased since the beginning of the AIDS epidemic, growing from 12 published cases before the epidemic to 80 published cases [1]. A relationship between the prophylactic use of aerosolised pentamidine and the development of extra-pulmonary pneumocystosis has been suggested [1]. The risk for extra-pulmonary dissemination of $P$. carinii in HIV-infected patients is estimated to range from 0.5 to $3 \%$ [1], but this rate is certainly underestimated because: (i) extra-pulmonary signs are infrequent and premortem diagnosis is hardly ever suspected even when such signs are present; (ii) autopsy studies are not systematically performed; and (iii) histological studies at necropsy only very rarely include a staining technique for $P$. carinii detection and DNA amplification has never been performed. The findings of the present experimental study suggest that the proliferation of the parasite is lower in extrapulmonary sites than in the lungs, where it is readily detectable in immunosuppressed rats by conventional techniques. Therefore, despite the small number of patients documented with extra-pulmonary dissemination of the parasite, the dissemination, in contrast to its clinical expression, is not necessarily a rare event. Clinical signs appear when $P$. carinii proliferation is massive, and can be detected by classical techniques.

A search for extra-pulmonary dissemination of $P$. carinii by stains has been performed in two experimental studies. Denis et al. [2] developed three models, i.e., rats and mice which received corticoster- oid treatment and SCID mice, all maintained in contact with $P$. carinii-infected mice. They observed extra-pulmonary dissemination, as noted in the present study, but the percentage of animals presenting with infection was lower than in the present study $(<50 \%)$. $\mathrm{Oz}$ et al. [3] also used conventional techniques to search for extra-pulmonary foci of $P$. carinii in 40 ferrets and 30 rats. These animals were immunosuppressed with dexamethasone, as in the present experiments, but were killed 7-8 weeks after the beginning of immunosuppression, instead of 12 weeks as in this study. $P$. carinii was not observed outside the lungs in rats, but it was found in $10 \%$ of the ferrets, in liver and spleen.

Other authors have used PCR. Evans et al. [13] searched for $P$. carinii DNA in the lung, liver, spleen and kidney of five immunosuppressed rats with pAZ102-E and pAZ102-H primers. $P$. carinii was found exclusively in the lung. However, the immunosuppressive regimen was shorter (6 weeks instead of 12) and less vigorous (hydrocortisone instead of dexamethasone) than the regimen used in the present study. The limit of detection of the DNA amplification was surprisingly high (590 organisms) even when a nested-PCR was used. Reddy et al. [14] amplified the dihydrofolate reductase (DHFR) gene to detect $P$. carinii in two rats immunosuppressed with dexamethasone. $P$. carinii was found in the liver, but not in the spleen or in the kidney. By following the amplification protocol described by Wakefield et al. [10], Furuta et al. [15] detected $P$. carinii in several organs of two out of five simian immunodeficiency virus (SIV)-infected monkeys, with positive results in as many as 14 extra-pulmonary sites in one monkey. More recently, with primers specific for the major surface glycoprotein (MSG) gene, Chary-Reddy et al. [16] found $P$. carinii DNA in several non-pulmonary tissues of 10 immunosuppressed rats, including kidney $(80 \%)$, liver $(90 \%)$ and spleen $(60 \%)$. Furthermore, reverse transcriptase-PCR analysis with DHFR primers 
demonstrated active transcription in these tissues and thus the presence of viable $P$. carinii organisms. These results are consistent with those of the present study although the immunosuppression protocol (methylprednisolone) and the amplification target were different.

The extra-pulmonary locations of $P$. carinii in the sentinel control rats lends support to our hypothesis that the extra-pulmonary dissemination from the lung may be a part of the normal progression of the disease. A search for $P$. carinii in non-pulmonary tissues in non-immunosuppressed animals housed near corticosteroid-treated rats has not been performed in the six studies cited above. Oz et al. [3], Reddy et al. [14] and Chary-Reddy et al. [16] did not find $P$. carinii in lung or in other organs in immunocompetent animals, but the authors did not specify whether these animals were housed near the $P$. carinii-infected animals. Furthermore, Chary-Reddy et al. [16] used special rats which were viral antibody-negative and $P$. carinii-free. No control animals were mentioned in the three other studies $[2,13,15]$. Thus the present findings in immunocompetent rats can be compared only with those of Sepkowitz et al. [12]. In their study $P$. carinii DNA was detected by PCR in lung tissue and in sera from non-immunocompromised sentinel rats that were exposed to $P$. carinii-infected rats. After a few weeks of isolation apart from the corticosteroidtreated rats, $P$. carinii DNA became undetectable in lung and in serum. The results of Sepkowitz et al. may reflect subclinical infection or a high level of lung colonisation and a low level parasitaemia after aerial transmission of $P$. carinii. The precise nature of the $P$. carinii DNA detected by PCR in blood is not known at the present time, but the fact that $P$. carinii can be isolated in cell line cultures from the blood of AIDS patients with pulmonary pneumocystosis [17] lends support to the hypothesis of viable circulating organisms. Thus the presence of $P$. carinii in the nonpulmonary tissues observed in immunocompetent sentinel rats in the present study may be the result of $P$. carinii dissemination via the haematogenous route. Further experiments are required to clarify this, in immunocompetent animals as well as in immunosuppressed animals. Extra-pulmonary sites are thus important, not so much because they generate pathogenic effects, but rather because they might be potential sources of development or relapse of pulmonary pneumocystosis.

The present study compared the usual stains used for the detection of $P$. carinii with PCR and demonstrated the usefulness of DNA amplification for detection of $P$. carinii in extra-pulmonary rat tissues. It is likely that the spread of $P$. carinii from the lungs to various organs is the natural outcome of infection in the host. This hypothesis has to be tested in man by a systematic search for $P$. carinii in extra-pulmonary tissues by PCR.

This study was supported by research grants from the 'Fondation pour la Recherche Médicale' and the 'Hospices Civils de Lyon.'

\section{References}

1. Telzak EE, Armstrong D. Extrapulmonary infection and other unusual manifestations of Pneumocystis carinii. In: Walzer PD (ed) New York, NY, Marcel Dekker, Inc. Pneumocystis carinii pneumonia, 2nd edn. 1994: 361-378.

2. Denis CM, Cailliez JC, Aliouat ME et al. Does Pneumocystis carinii remain infectious in the bloodstream? J Euk Microbiol 1994; 41: 86S.

3. Oz HS, Hugues WT, Vargas SL. Search for extrapulmonary Pneumocystis carinii in an animal model. J Parasitol 1996; 82: $357-359$.

4. Wakefield AE, Pixley FJ, Banerji S et al. Detection of Pneumocystis carinii with DNA amplification. Lancet 1990; 336: $451-453$.

5. Kitada K, Oka S, Kimura S et al. Detection of Pneumocystis carinii sequences by polymerase chain reaction: animal models and clinical application to non invasive specimens. $J$ Clin Microbiol 1991; 29: 1985-1990.

6. Schluger, N. Goldwin T, Sepkowitz K et al. Application of DNA amplification to pneumocystosis: presence of serum Pneumocystis carinii DNA during human and experimentally induced Pneumocystis carinii pneumonia. J Exp Med 1992; 176: $1327-1333$

7. Olsson M, Elvin K, Löfdahl S, Linder E. Detection of Pneumocystis carinii DNA in sputum and bronchoalveolar lavage samples by polymerase chain reaction. $J$ Clin Microbiol 1993; 31: 221-226.

8. Leibovitz E, Pollack H, Moore T et al. Comparison of PCR and standard cytological staining for detection of Pneumocystis carinii from respiratory specimens from patients with or at high risk for infection by human immunodeficiency virus. $J$ Clin Microbiol 1995; 33: 3004-3007.

9. Leigh TR, Gazzard BG, Rowbottom A, Collins JV. Quantitative and qualitative comparison of DNA amplification by PCR with immunofluorescence staining for diagnosis of Pneumocystis carinii pneumonia. J Clin Pathol 1993; 46: 140-144.

10. Wakefield AE, Pixley FJ, Banerji $\mathrm{S}$ et al. Amplification of mitochondrial ribosomal RNA sequences from Pneumocystis carinii DNA of rat and human origin. Mol Biochem Parasitol $1990 ;$ 43: $69-76$.

11. Kwok S, Higuchi R. Avoiding false positives with PCR. Nature 1989; 339: 237-238.

12. Sepkowitz K, Schluger N, Godwin T, Armstrong D, Cerami A, Bucala R. DNA amplification in experimental pneumocystosis: characterization of serum Pneumocystis carinii DNA and potential carrier states. J Infect Dis 1993; 168: 421-426.

13. Evans R, Joss AWL, Pennington TH, Ho-Yen DO. The use of a nested polymerase chain reaction for detecting Pneumocystis carinii from lung and blood in rat and human infection. $J$ Med Microbiol 1995; 42: 209-213.

14. Reddy LV, Zammit C, Schuman P, Crane LR. Detection of Pneumocystis carinii in a rat model of infection by polymerase chain reaction. Mol Cell Probes 1992; 6: 137-143.

15. Furuta T, Fujita M, Mukai R. Severe pulmonary pneumocystosis in simian acquired immunodeficiency syndrome induced by simian immunodeficiency virus: its characterization by the polymerase chain reaction method and failure of experimental transmission to immunodeficient animals. Parasitol Res 1993; 79: $624-628$

16. Chary-Reddy S, Graves DC. Identification of extrapulmonary Pneumocystis carinii in immunocompromised rats by PCR. $J$ Clin Microbiol 1996; 34: 1660-1665.

17. Contini C, Mastrantoni S, Romani R, Cultrera R, Delia S. Evidence of Pneumocystis carinii in cell line cultures infected with peripheral blood mononuclear cells isolated from AIDS patients with $P$. carinii pneumonia. J Med Microbiol 1995; 42: 394-398. 